\title{
Efficacy of fibre additions to flatbread flour mixes for reducing post-meal glucose and insulin responses in healthy Indian subjects
}

\author{
Hanny M. Boers ${ }^{1 *}$, Katrina MacAulay ${ }^{2}$, Peter Murray $^{2}$, Rajendra Dobriyal ${ }^{3}$, David J. Mela ${ }^{1}$ \\ and Maria A. M. Spreeuwenberg ${ }^{1}$ \\ ${ }^{1}$ Unilever Discover RED Vlaardingen, PO Box 114, 3130 AC, Vlaardingen, The Netherlands \\ ${ }^{2}$ Unilever Discover RED Colworth, Colworth Science Park, Sharnbrook MK44 1LQ, UK \\ ${ }^{3}$ Unilever Clinicals RED Bangalore, 64 Main Road, Whitefield, Bangalore, 560 066, India \\ (Submitted 15 July 2016 - Final revision received 15 December 2016 - Accepted 24 January 2017)
}

\section{Abstract}

The incidence of type 2 diabetes mellitus (T2DM) is increasing worldwide, including in developing countries, particularly in South Asia. Intakes of foods generating a high postprandial glucose (PPG) response have been positively associated with T2DM. As part of efforts to identify effective and feasible strategies to reduce the glycaemic impact of carbohydrate-rich staples, we previously found that addition of guar gum (GG) and chickpea flour (CPF) to wheat flour could significantly reduce the PPG response to flatbread products. On the basis of the results of an exploratory study with Caucasian subjects, we have now tested the effect of additions of specific combinations of CPF with low doses of GG to a flatbread flour mix for their impacts on PPG and postprandial insulin (PPI) responses in a South-Asian population. In a randomised, placebo-controlled full-cross-over design, fifty-six healthy Indian adults consumed flatbreads made with a commercial flatbread mix (100\% wheat flour) with no further additions (control) or incorporating $15 \%$ CPF in combination with 2,3 or $4 \%$ GG. The flatbreads with $\mathrm{CPF}$ and 3 or $4 \%$ GG significantly reduced PPG (both $\geq 15 \%$ reduction in positive incremental AUC, $P<0.01$ ) and PPI (both $\geq 28 \%$ reduction in total AUC, $P<0.0001)$ compared with flatbreads made from control flour. These results confirm the efficacy and feasibility of the addition of CPF with GG to flatbread flour mixes to achieve significant reductions in both PPG and PPI in Indian subjects.

Key words: Atta: Viscous fibres: Legume flours: Glycaemic responses: Insulin responses: Appetite

There is a growing global epidemic of type 2 diabetes mellitus (T2DM), especially in developing countries such as India ${ }^{(1)}$. Consequently, there is considerable public health and consumer interest in taking steps to reduce this risk. Continuous exposures to higher postprandial glucose (PPG) and postprandial insulin (PPI) responses are believed to be detrimental to health, contributing towards an increased risk for (pre)diabetes ${ }^{(2)}$. There is a wealth of literature showing that reducing PPG (by slowing rates of digestion and reducing bioavailability) ${ }^{(3)}$ has benefits for reducing the progression from pre-diabetes to $\mathrm{T}_{2} \mathrm{DM}^{(4-6)}$ and the risk for $\mathrm{CVD}^{(7,8)}$.

A lower PPI requirement may also be beneficial in the short and longer term. Consistent with this, the European Food Safety Authority has recognised that the reduction of postprandial glycaemia may be a beneficial physiological effect, but only if PPI is not disproportionally increased ${ }^{(9)}$.

Because of their frequent and consistent use, carbohydraterich staple foods are interesting candidates for reducing PPG and PPI exposures ${ }^{(10)}$. Wheat-based flatbreads and rice are the two most common carbohydrate-rich staple foods in Southeast Asia ${ }^{(11)}$, making them important contributors to the daily glycaemic load. Flatbreads are usually prepared at home from a commercially made whole-wheat flour mix ('atta'). Chickpea flour (CPF) and bran-fibre flatbreads are especially advised for subjects with T2DM for whom rice is considered less desirable because of its high glycaemic index ${ }^{(12)}$. Therefore, commercially viable, efficacious routes to further reduce the PPG response to flatbreads are of interest.

Soluble viscous fibres can lower $\mathrm{PPG}^{(13)}$ by delaying gastric emptying ${ }^{(14,15)}$ and inhibiting the propulsive and mixing effects in the intestine ${ }^{(16,17)}$. In addition, legume flours such as CPF are known to give a lower plasma glucose response than wheat flours $^{(18)}$. Previous research has shown that soluble viscous fibres (viz. $\beta$-glucan, psyllium and fenugreek) with or without legume flour can lower the $\mathrm{PPG}^{(12,19,20)}$ or $\mathrm{PPI}^{(12)}$ of flatbreads.

The emphasis of this study was on commercially feasible products. We ${ }^{(21)}$ previously selected and tested various additions of guar gum (GG), konjac or CPF to flatbreads in

Abbreviations: BF, barley flour; $C_{\max }$, maximum observed glucose response; CPF, chickpea flour; GG, guar gum; PPG, postprandial glucose; PPI, postprandial insulin; $+\mathrm{iAUC}_{2 \mathrm{~h}}$, positive incremental $\mathrm{AUC} 2 \mathrm{~h}$; tAUC, total AUC.

*Corresponding author: H. M. Boers, fax +31 10460 5993, email hanny.boers@unilever.com 
Caucasian subjects. In that study, the composition with $4 \%$ GG in combination with $15 \% \mathrm{CPF}$ resulted in the largest absolute reduction in PPG and could therefore be considered a 'positive control' for future studies. However, GG is expensive, and product development work showed that it created a poor product with respect to sensory attributes (dough handling, texture, aroma). Lower amounts of GG (2 or 3\% GG) in combination with $15 \% \mathrm{CPF}$ and additions of 3 and $5 \%$ (respectively) barley flour (BF) made more acceptable and affordable products $^{(22)}$, and these have been used in this trial, alongside the $4 \% \mathrm{GG}$ and $15 \% \mathrm{CPF}$. The reason for including different amounts of $\mathrm{BF}$ is that higher amounts of $\mathrm{GG}$ benefit from more $\mathrm{BF}$ in helping to mask the GG flavour and make a more consumer-acceptable product. The present study aims to confirm the efficacy of the combinations with 2 and $4 \%$ GG, which were reported in the previous study (both with a significant effect), and to further extend this work to the Indian population. The level of added BF contains only small amounts of viscous fibres ( $(\leq 250 \mathrm{mg})$ of barley $\beta$-glucan) and is unlikely to influence efficacy ${ }^{(23)}$. The control product was a market standard commercial wheat flour-based product ( $8 \%$ dietary fibre), whereas the test products were based on an alternative, commercially available, high-fibre atta flour (with 5\% wheat bran included; $12 \%$ dietary fibre). In our previous study, we did not see any effect on PPG between the market standard and highfibre atta products ${ }^{(21)}$. For the present study, the higher fibre atta was deemed a more appropriate (commercially realistic) platform for further additions of fibre, and these complete formulations were then compared with the usual, 'standard' atta product.

The objectives of this study were to identify one or more flour compositions that give a significant reduction in PPG and PPI after consumption, relative to the market standard product. Exploratory objectives were to estimate the maximum observed glucose response $\left(C_{\max }\right)$, the time at which the $C_{\max }$ was reached $\left(T_{\max }\right)$ and slope to $C_{\max }$ and glucose and insulin concentrations at $3 \mathrm{~h}$. As soluble viscous fibres and CPF are also claimed to increase satiety ${ }^{(24,25)}$, an additional exploratory objective was to assess possible effects on appetite-related and mood parameters. To our knowledge, this is the first study testing such commercially feasible combinations of soluble viscous fibres and legume flour in flatbreads on the combined PPG and PPI response in a Southeast Asian population.

\section{Methods \\ Participants}

A total of eighty-seven healthy South-Asian subjects were recruited locally for screening from an existing database of potential participants of Lambda Therapeutic Research, which executed the study. Subjects who met all the inclusion criteria and had none of the exclusion criteria were considered for participation (online Supplementary Table S1). The study was conducted according to the principles of Good Clinical Practice, the Declaration of Helsinki (2008) and according to applicable local laws and regulations concerning studies conducted on human subjects. Ethical approval was obtained from
Ethical Committee-Aditya. Each participant provided written informed consent for the study.

\section{Experimental design}

This study used a double-blind, randomised, controlled, fullcross-over (within-subject) design. Treatment orders were balanced according to a Williams-type design, and a randomised schedule for allocation to treatment orders was generated with SAS software (version 9.2; SAS Institute Inc.) by a statistician not involved with subject contact or subsequent data analyses. All subjects involved in the study were blinded as to the nature of the test products. Subjects attended the initial screening day, followed by $4 \mathrm{~d}$ of test, at least 1 week apart. Participants were instructed to minimise changes in their habitual diet and activity during the study period. On the day prior to each test day subjects were instructed to refrain from physical activity and alcohol consumption and to consume a standardised evening meal. All participants fasted overnight (from 20.00 hours until consumption of the test product) but were allowed to drink water ad libitum. Participants were housed at the test centre the evening before the study day. Between 07.30 and 10.00 hours on each test day, subjects consumed four freshly made flatbreads ( $100 \mathrm{~g}$ flour total) with $250 \mathrm{ml}$ water as breakfast, and completed this within a $15-\mathrm{min}$ period at every visit at the same time and day of the week. They were allowed to drink up to $150 \mathrm{ml}$ water every subsequent hour, to be consumed after venous blood drawings and self-reported appetite and mood ratings. The volume of water consumed was registered.

\section{Test product and preparation}

The three wheat flour-based test products constituted a total of $100 \mathrm{~g}$ (uncooked flour weight) of an existing commercial, fibreenriched flatbread high-fibre flour (Annapurna; Hindustan Unilever Ltd), comprising whole-wheat flour plus 5\% bran and the incorporation of $15 \mathrm{~g} \mathrm{CPF}, 2,3$ or $4 \mathrm{~g} \mathrm{GG}$ and 3 or $5 \mathrm{~g} \mathrm{BF} /$ $100 \mathrm{~g}$ in combinations shown in Table 1. A 'market standard', Annapurna Atta (100\% wheat flour), was used as the control.

All experimental flour mixes were formulated at the pilot plant of Unilever R\&D, Bangalore, India, and flatbreads were prepared fresh at the test site. For each single test serving, $100 \mathrm{~g}$ flour was kneaded to a soft and uniform consistency with the addition of approximately $77 \mathrm{ml}$ water and allowed to rest for $30 \mathrm{~min}$, and then divided into four equal balls of $40 \mathrm{~g}$ each and rolled to $2-3 \mathrm{~mm}$ thickness. More water was added and absorbed when fibres or legume flour was incorporated (see Table 1). The flatbreads were subsequently baked and kept warm until consumption within 30 min of cooking.

\section{Blood collection and glucose and insulin measurements}

Venous blood was collected in tubes containing sodium fluoride for plasma glucose analysis and in plain tubes (without any additive) for serum insulin samples. Baseline samples were collected at $-15 \mathrm{~min}$ (two baseline measurements) prior to the test meals, followed by samples at 15, 30, 45, 60, 90, 120 and 
Table 1. Composition of test flatbreads + carbohydrates (carbs) and dietary fibre (g) and water (w/w \%)

\begin{tabular}{lccc}
\hline Compositions & Total available carbs $(\mathrm{g})$ & Total dietary fibre $(\mathrm{g})$ (AOAC 2009.8) & Water (w/w \%) \\
\hline $100 \mathrm{~g}$ market standard flour & 65 & 8 & 37.2 \\
$80 \mathrm{~g} \mathrm{HFF}+15 \mathrm{~g} \mathrm{CPF}+2 \mathrm{~g} \mathrm{GG}+3 \mathrm{~g} \mathrm{BF \ddagger}$ & 56 & 16 & 40.6 \\
$77 \mathrm{~g} \mathrm{HFF}+15 \mathrm{~g} \mathrm{CPF}+3 \mathrm{~g} \mathrm{GG}+5 \mathrm{~g} \mathrm{BF}$ & 54 & 17 & $42 \cdot 2$ \\
$81 \mathrm{~g} \mathrm{HFF}+15 \mathrm{~g} \mathrm{CPF}+4 \mathrm{~g} \mathrm{GG}$ & 53 & 18 & $42 \cdot 4$ \\
\hline
\end{tabular}

AOAC, Association of Official Analytical Chemists; HFF, high-fibre flour; CPF, chickpea flour; GG, guar gum; BF, barley flour.

* Chickpea flour (Avent Agro Pvt Ltd).

† Guar gum (Ace Gum Industries Pvt Ltd); viscosity cold 1\% in water, measured by a Brookfield RVF viscometer 20-RPM Spindle no. 4 (Brookfield Engineering Laboratories, Inc.), at $30 \mathrm{~min}: 4500 \mathrm{mPa}$ s, at $2 \mathrm{~h} 5400 \mathrm{CPS}$ and $24 \mathrm{~h}: 5500 \mathrm{mPa}$ s.

$\ddagger$ BF (Cardin Healthcare Pvt Ltd).

$180 \mathrm{~min}$ postprandially. Two consecutive samples were collected at each time-point for plasma glucose and serum insulin analyses. All serum samples were centrifuged ( $192 \mathbf{g}$ for $10 \mathrm{~min}$ at $\left.4^{\circ} \mathrm{C}\right)$ prior to immediate analysis or storage at $-20^{\circ} \mathrm{C}$. Plasma glucose concentrations were measured on VITROS ${ }^{\circledR}$ 5,1 FS, Ortho Clinical Diagnostics (Johnson \& Johnson) (intra(within-) day \%CV: 0.6\% and between- (inter-) day \%CV: 0.7\%). Insulin was measured using an immulite $1000^{\circledR}$ analyzer (Siemens Diagnostics) (intra- (within-) day \%CV: $2.6 \%$ and between- (inter-) day \%CV: $4 \cdot 3 \%$ ).

\section{Measurement of appetite and mood}

Self-ratings of appetite feelings and mood ('are you feeling hungry?', 'do you desire to eat?', 'are you feeling energetic?' and 'are you feeling happy and contented?') were made at baseline (before consumption) and 25, 40 and $135 \mathrm{~min}$ postprandially. The questions were asked in a language that the subjects understood. These were scored on $100 \mathrm{~mm}$ visual analogue scales anchored at the low and high end with 'not at all' and 'extremely',(26).

\section{Statistical methods}

The primary outcome variable was positive incremental $\mathrm{AUC}_{2} \mathrm{~h}$ $\left(+\mathrm{iAUC}_{2} \mathrm{~h}\right)$ : that is, the area of PPG response lying above the baseline concentration. A power calculation indicated that a minimum of forty-four subjects would be required to test for the significance of a $40 \mathrm{mmol} \times \mathrm{min} / 1$ difference in $\mathrm{PPG}+\mathrm{iAUC}_{2 \mathrm{~h}}$ for each test product relative to the control. This assumed an SD of 50 (based on a previous study in the $\mathrm{UK}^{(21)}$ adjusted for venous $v$. capillary blood), at $\alpha 0.05$ and $\beta 0.90$, where $\alpha$ is conserved over the three comparisons with the control by Dunnett's test. Considering the Williams design for four treatments and the experience of dropouts at the test site, twelve additional subjects were included to make the initial sample size of $n 56$.

The $+\mathrm{iAUC}_{2 \mathrm{~h}}$ was calculated using the trapezium rule, and linear interpolation was used between time points where PPG crossed the baseline value to establish the time of crossing. Statistical comparisons were made using a mixed-model ANOVA, with subject as a random effect, product as a fixed effect and baseline (fasting score) as a covariate. The order of product testing, sex and body weight were all included as covariates. Comparisons were only made between the control and other test products, and Dunnett's test was used to adjust the multiple comparisons to an overall significance level of 0.05. All analyses were performed with SAS version 9.2. The secondary variable was total $\mathrm{AUC}_{2 \mathrm{~h}}\left(\mathrm{tAUC}_{2 \mathrm{~h}}\right)$ for serum insulin. Exploratory variables included mean value at $3 \mathrm{~h}$ for serum insulin, maximum post-meal plasma glucose concentration $\left(C_{\max }\right)$, time when this was reached $\left(T_{\max }\right)$, and mean value at $3 \mathrm{~h}$ for plasma glucose, $\mathrm{tAUC}_{2} \mathrm{~h}$ glucose and selfreported scores on the two appetite- and two mood-related measures. The AUC for appetite- and mood-rating scales were calculated using the trapezium rule and expressed as the original scale units by dividing with the length of time measured. There were no pre-planned statistical analyses of exploratory measures and therefore only descriptive statistics are presented for these.

\section{Results}

\section{Subject baseline characteristics}

From an initial eighty-seven subjects screened for participation, fifty-six were enrolled and fifty (twenty-five male and twentyfive female) completed the study, with all dropouts occurring prior to the start or after the first test session (Fig. 1). The baseline characteristics of participants are shown in Table 2(a) and separately by sex in Table 2(b). Differences between results of the intention to treat (ITT) and per protocol (PP) analysis were small regarding both effect sizes and statistical significance. However, the PP results are shown and discussed here, as the current study was a proofof-principle study (study of efficacy). For completeness and transparency, the ITT data are available in the online Supplementary material.

\section{Postprandial plasma glucose concentrations}

The PPG response curves are shown in Fig. 2, percentage differences in plasma glucose $+\mathrm{iAUC}_{2 \mathrm{~h}} v$. control are shown in Fig. 3, and the absolute values and percentage difference are given in Table 3. All three test products resulted in a reduction in the postprandial $+\mathrm{iAUC}_{2} \mathrm{~h}$, relative to the control market standard flatbread, and this difference was statistically significant for samples containing 3 and $4 \%$ GG (both $P<0 \cdot 01$ ). The data suggest a general dose-response reduction in $+\mathrm{iAUC}_{2 \mathrm{~h}}$ with 2,3 and $4 \%$ GG relative to control; however, this was not tested statistically as no comparisons between treatments were made. 


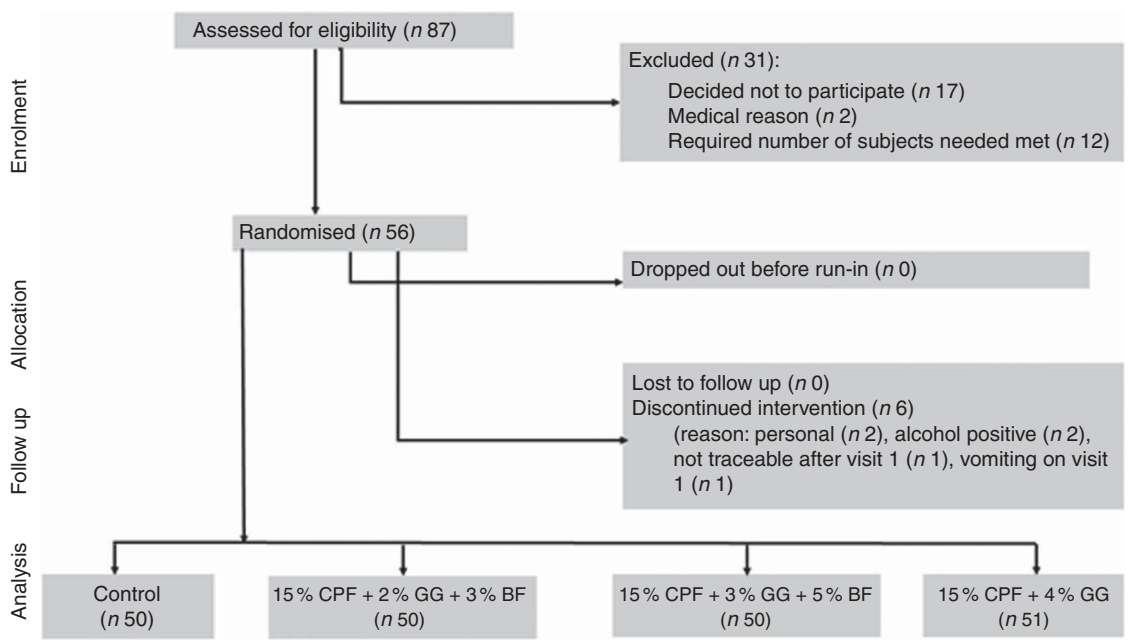

Fig. 1. Flow diagram of participants throughout the study. CPF, chickpea flour; GG, guar gum; BF, barley flour.

Table 2a. Subject baseline demographic characteristics (Mean values and standard errors of the mean)

\begin{tabular}{lrr}
\hline & Mean & SEM \\
\hline Age (years) & 29.16 & 0.71 \\
Sex $(\%$ male) & 53.57 & \\
Height $(\mathrm{m})$ & 1.59 & 0.01 \\
Body weight $(\mathrm{kg})$ & 52.57 & 0.81 \\
BMl $\left(\mathrm{kg} / \mathrm{m}^{2}\right)$ & 20.77 & 0.20 \\
Fasting blood glucose $(\mathrm{mmol} / \mathrm{l})$ & 4.89 & 0.03 \\
\hline
\end{tabular}

Table $\mathbf{2 b}$. Subject baseline demographic characteristics by sex (Mean values and standard errors of the mean)

\begin{tabular}{lrrrrr}
\hline & \multicolumn{2}{c}{ Male $(n$ 30) } & & \multicolumn{2}{c}{ Female $(n$ 26 $)$} \\
\cline { 2 - 3 } & Mean & SEM & & Mean & SEM \\
\hline Age (years) & 27.33 & 1.05 & & 31.27 & 0.75 \\
Height $(\mathrm{m})$ & 1.66 & 0.01 & & 1.51 & 0.01 \\
Body weight $(\mathrm{kg})$ & 56.21 & 0.94 & & 48.36 & 0.76 \\
BMl $\left(\mathrm{kg} / \mathrm{m}^{2}\right)$ & 20.48 & 0.28 & & 21.11 & 0.27 \\
Fasting blood glucose (mmol/l) & 4.87 & 0.05 & & 4.90 & 0.04 \\
\hline
\end{tabular}

\section{Postprandial serum insulin concentrations}

Data for serum insulin (tAUC) can be found in Fig. 4 and 5 and Table 4. All three test flatbreads significantly and markedly lowered postprandial total PPI tAUC $_{2} \mathrm{~h}$ compared with the control flatbread $(P<0.0001$ for all). In addition, the data suggest a dose-response effect, but this was not tested statistically.

\section{Exploratory outcomes}

tAUC, $C_{\max }, T_{\max }, 3 \mathrm{~h}$ plasma glucose data and slope to $C_{\max }$ data are shown in the online Supplementary Tables S2(a) and (b). All treatments resulted in lower mean $C_{\max }$ and slope to $C_{\max }$ values compared with the control, whereas $T_{\max }$ and plasma glucose at $3 \mathrm{~h}$ were in general little different from that of the control. Data for serum insulin (+iAUC) can be found in the online Supplementary Fig. S1. Serum insulin concentrations at $3 \mathrm{~h}$ were consistently lower for all three experimental products relative to the control (see online Supplementary Table S3). No reliable effect of the experimental treatments could be discerned for the appetite or mood measures (online Supplementary Fig. S2(a) and (b), S3(a) and (b)).

\section{Adverse events}

One adverse event (AE) was reported during the conduct of the study. A female subject vomited after consuming the product with $4 \%$ GG and $15 \% \mathrm{CPF}$ on her 1 st test day. This AE was resolved within $30 \mathrm{~min}$ without requiring any medical treatment, and the subject was excluded from the rest of the study.

\section{Discussion}

This study clearly shows that a commercially feasible, high-fibre formulation including the addition of small amounts of GG in combination with CPF lowers the PPG and PPI responses to flatbreads in an Asian population, relative to a commercial 'market standard' flour mix. This finding builds on the outcome of our initial exploratory study with Caucasian subjects ${ }^{(21)}$ in which four combinations of flour and fibre mixes decreased the $+\mathrm{iAUC}$ by $>30 \%$. The composition with $4 \%$ GG and $15 \% \mathrm{CPF}$ from that study was carried along as 'positive control', as it resulted in the largest absolute reduction in $\mathrm{PPG}^{(21)}$. However, this amount of GG has adverse impacts on cost, dough handling and aroma, and therefore lower levels of GG were needed. Our research is in line with reports demonstrating that other staple flatbread foods in India (chapattis, naan, rotis) containing dietary fibres and/or legume flours lower the PPG response after a meal ${ }^{(12,19,20)}$. A novel result is the demonstrated efficacy both on PPG and PPI reduction of the combination of CPF with lower levels of GG and $\mathrm{BF}$ in Asians, as efficacy of this combination previously had been shown only in Caucasians and only on PPG reduction ${ }^{(21)}$. This suggests the potential for affordable, efficacious formulations with lower levels of GG. 
Although there was a trend for a dose-response effect observed for the additions of 2,3 and $4 \%$ of GG alone on PPG $\left(+\mathrm{iAUC}_{2} \mathrm{~h}\right)$, the effects were statistically significant only for 3 and $4 \%$ GG compared with the control product. It seems that addition of $2 \%$ GG may be too low to have a consistently meaningful effect related to the effect size on PPG in this food format. Brennan ${ }^{(27)}$ calculated that $2.5 \%$ GG lowered the predicted glycaemic index (PGI) in bread by $4 \%$, whereas $5 \%$ GG reduced the PGI by $13 \%$. There was also an indication of a dose-response effect on the $C_{\max }$. Other studies have shown the efficacy of GG (ranging from 3.8 to $14.8 \mathrm{~g} \mathrm{GG}$ ) incorporated into bread for lowering PPG in Caucasians, and these showed a greater reduction in PPG than the outcomes here ${ }^{(28-31)}$.

GG has been found to exhibit viscous characteristics throughout gastric and small intestinal simulation ${ }^{(27)}$, leading to reductions in the rate of gastric emptying and starch digestion and absorption in the intestine, resulting in a lower PPG and insulin response ${ }^{(13)}$. GG and some other fibres can even directly inhibit digestive enzymes ${ }^{(32,33)}$. On the product level, viscous fibres can also alter the rheological and/or microstructural properties of the food, resulting in reduced ability of the starch to gelatinise during cooking ${ }^{(34)}$. Scanning electron microscopy has shown that GG included in bread forms a dense, continuous network in which protein, dietary fibre and starch

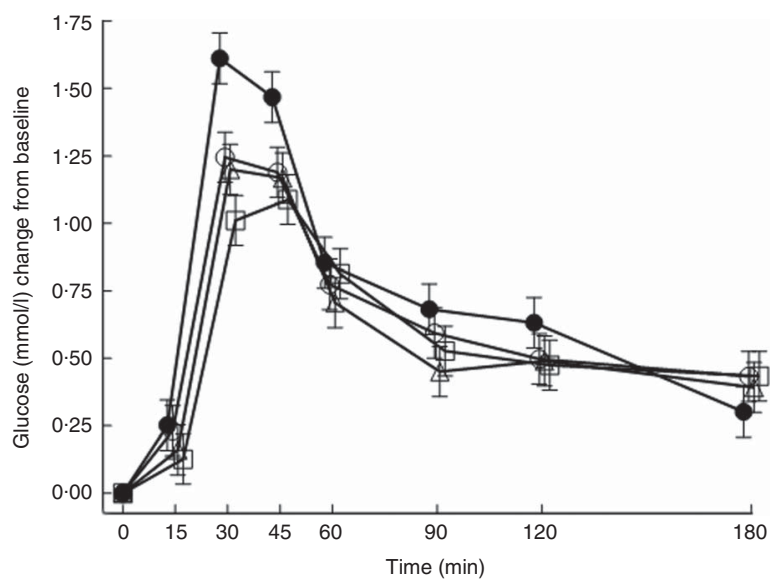

Fig. 2. Effect of flatbread consumption with different amounts of guar gum (GG) (2-4\%) and $15 \%$ chickpea flour (CPF) on postprandial plasma glucose. Values are means and standard errors of the mean. Market standard; $\bigcirc$, high-fibre flour (HFF) $+15 \mathrm{~g} \mathrm{CPF}+2 \mathrm{~g} \mathrm{GG}+3 \mathrm{~g}$ barley flour $(\mathrm{BF}) ; \triangle, \mathrm{HFF}+15 \mathrm{~g}$ $\mathrm{CPF}+3 \mathrm{~g} \mathrm{GG}+5 \mathrm{~g} \mathrm{BF} ; \square, \mathrm{HFF}+15 \mathrm{~g} \mathrm{CPF}+4 \mathrm{~g} \mathrm{GG}$. are embedded ${ }^{(27)}$. This dense network was found to be retained after $300 \mathrm{~min}$ of in vitro digestion, while the majority of the structure of a control bread (without GG) had been degraded ${ }^{(27)}$.

It is possible that the small amounts of added $\mathrm{BF}$ might also have influenced the outcomes of this study. However, it appears that $4 \mathrm{~g}$ of processed barley $\beta$-glucan is the minimum required dosage to get a meaningful PPG-lowering effect with respect to effect size ${ }^{(23)}$. The dosages used in this study (3 and $5 \mathrm{~g} \mathrm{BF}$ containing $5 \%(\leq 250 \mathrm{mg})$ of barley $\beta$-glucan $)$ are therefore very unlikely to have meaningfully affected the efficacy.

It is also possible that the addition of $\mathrm{CPF}$ contributed to the lower PPG. Replacement of wheat flour by CPF can lower the PPG because of its higher content of resistant starch ${ }^{(35)}$ and high concentration of slowly digestible $\operatorname{starch}^{(36)}$. Zafar et al. ${ }^{(37)}$ showed that supplementation of whole-wheat bread with at least $35 \% \mathrm{CPF}$ significantly reduced the glycaemic response of whole-wheat bread, whereas Johnson et al. ${ }^{(38)}$ observed this in white bread.

A lower PPI requirement may also be beneficial in the short and longer term. In the short term, a lower insulin response prevents hypoglycaemia and inappropriate increases of free fatty acids and stress hormone concentrations ${ }^{(39-41)}$, which are often seen during the late postprandial period after consumption of refined carbohydrates ${ }^{(42)}$. Regular consumption of diets with a low PPI response - for example, rye-pasta diets - may also benefit individuals with impaired first-phase insulin secretion (first $10-30 \mathrm{~min}$ ) by allowing $\beta$-cell function to recover, leading to improved pancreatic $\beta$-cell function in the long term ${ }^{(43)}$. Higher PPI and fasting insulin concentrations are positively associated with CVD risk factors such as blood pressure, total cholesterol and LDL-cholesterol ${ }^{(44)}$. In addition, insulin resistance and postprandial hyperinsulinaemia are related to impaired arterial relaxation, which is an independent predictor of $\mathrm{CVD}^{(45)}$.

We found that all three test flatbread flour mixes significantly and markedly reduced PPI levels relative to the control. The reason for the lower PPI response of the test flatbreads with fibre and flour mix is probably a slower glucose absorption in the blood, resulting in a reduced stimulation of the enteroinsular axis, notably the incretin gastric inhibitory polypeptide (GIP), the secretion of which is directly related to the rate and site of absorption of glucose ${ }^{(46-48)}$. It has also been observed that GG in bread directly lowered the GIP response ${ }^{(29)}$.

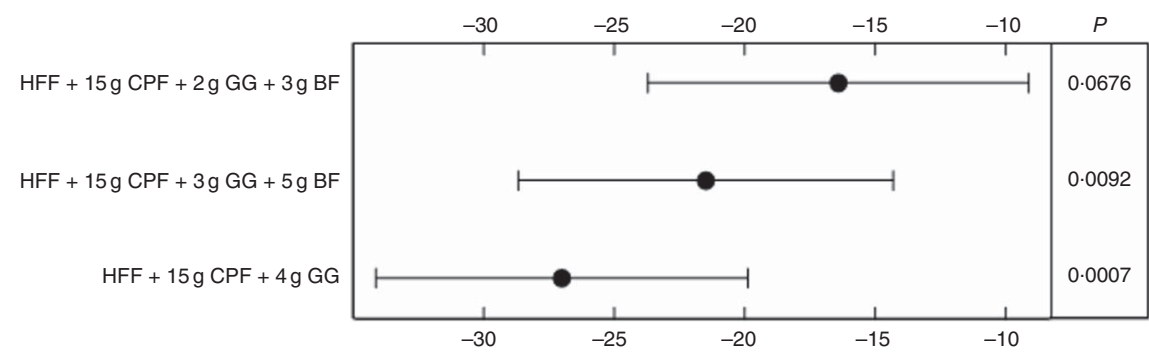

Fig. 3. Percentage change (mean values and standard errors of the mean) in postprandial glucose (positive incremental $A U C_{2 h}\left(+i A U C_{2} h\right)$ ) of flatbreads with different amounts of guar gum (GG) (2-4\%) and $15 \%$ chickpea flour (CPF) and $P$ value relative to the control. HFF, high-fibre flour; BF, barley flour. 
Table 3. Glucose positive incremental $\mathrm{AUC}_{0-2 h}\left(+\mathrm{iAUC}_{0-2 \mathrm{~h}}\right)$ (per protocol data) (Mean absolute and percentage difference from control; mean values +/- SEM)

\begin{tabular}{|c|c|c|c|c|}
\hline & \multicolumn{4}{|c|}{ Glucose + iAUC ${ }_{0-2 h}$ (control: 104.9 mmol/l.min) } \\
\hline & \multicolumn{2}{|c|}{ Absolute (mmol/l.min) } & \multicolumn{2}{|c|}{ Difference $(\%)$} \\
\hline & Mean & SEM & Mean & SEM \\
\hline \multicolumn{5}{|l|}{ Flatbread compositions } \\
\hline $80 \mathrm{~g} \mathrm{HFF}+15 \mathrm{~g} \mathrm{CPF}+2 \mathrm{~g} \mathrm{GG}+3 \mathrm{~g} \mathrm{BF}$ & -17.21 & 7.66 & -16.4 & 7.30 \\
\hline $77 \mathrm{~g} \mathrm{HFF}+15 \mathrm{~g} \mathrm{CPF}+3 \mathrm{~g} \mathrm{GG}+5 \mathrm{~g} \mathrm{BF}$ & $-22.54^{*}$ & 7.53 & $-21.5^{\star}$ & 7.18 \\
\hline $81 \mathrm{~g} \mathrm{HFF}+15 \mathrm{~g} \mathrm{CPF}+4 \mathrm{~g} \mathrm{GG}$ & $-28.33^{*}$ & 7.49 & $-27.0^{*}$ & 7.13 \\
\hline
\end{tabular}

HFF, high fibre; CPF, chickpea flour; GG, guar gum; BF, barley flour. Statistically significant: ${ }^{\star} P<0.01$.

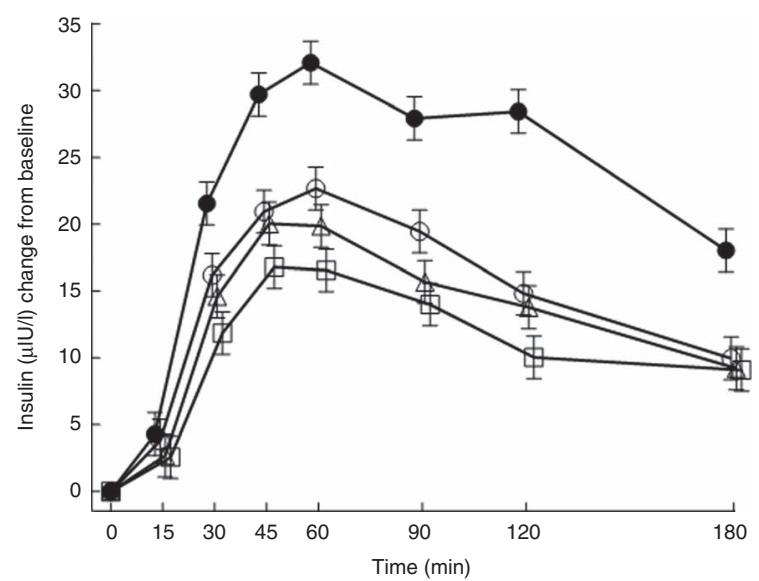

Fig. 4. Effect of flatbread consumption with different amounts of guar gum (GG) $(2-4 \%)$ and $15 \%$ chickpea flour (CPF) on postprandial serum insulin. Values are means and standard errors of the mean. Market standard; $O$, high-fibre flour (HFF) $+15 \mathrm{~g} \mathrm{CPF}+2 \mathrm{~g} \mathrm{GG}+3 \mathrm{~g}$ barley flour (BF); $\triangle, \mathrm{HFF}+15 \mathrm{~g}$ $\mathrm{CPF}+3 \mathrm{~g} \mathrm{GG}+5 \mathrm{~g} \mathrm{BF} ; \square, \mathrm{HFF}+15 \mathrm{~g} \mathrm{CPF}+4 \mathrm{~g} \mathrm{GG}$.
Khawaja et $a l^{(12)}$ showed that flatbread containing bran resulted in a lower PPI response than flatbread without bran (19.3 and 8.6 g dietary fibre, respectively) in healthy Asians as well as in Asians with T2DM, but our products did contain lower amounts of bran ( 8 and $12 \mathrm{~g}$ for the market standard and high fibre, respectively). Previous reports have demonstrated that supplementation of bread with GG results in a substantially lower PPI ${ }^{(28-30,49)}$.

The contribution of CPF to the reduced PPI is not clear, as studies on the effect of chickpeas on PPI have yielded mixed results. Nestel et $a l^{\left({ }^{(36)}\right.}$ showed in healthy subjects a serum insulin reduction of $55 \%$ at 30 and $60 \mathrm{~min}$ after consumption of mashed chickpeas compared with a wheat-based meal ${ }^{(36)}$, whereas Johnson et al. ${ }^{(38)}$ reported an insulin increase of $+\mathrm{iAUC}$ of $32 \%$ when $24 \%$ of the wheat flour was replaced by $\mathrm{CPF}^{(38)}$. This discrepancy can be explained by the fact that fine grinding of legumes (as is the case for CPF) disrupts the cell structure and renders starch more readily accessible for digestion $^{(50)}$.

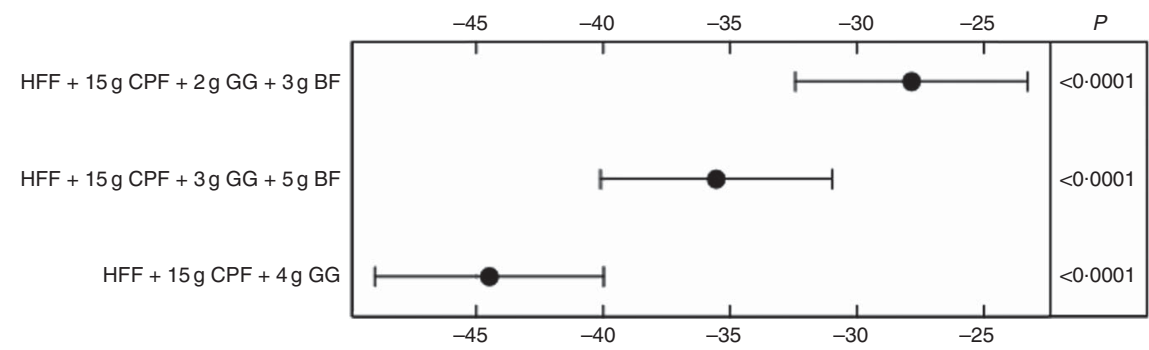

Fig. 5. Percentage change (mean values and standard errors of the mean) in postprandial insulin (total $A \cup C_{2 h}\left(t A \cup C_{2 h}\right)$ ) of flatbreads with different amounts of guar gum (GG) (2-4\%) and $15 \%$ chickpea flour (CPF) and $P$ value relative to the control. HFF, high-fibre flour; BF, barley flour.

Table 4. Insulin total $\mathrm{AUC}_{0-2 h}\left(\mathrm{t} A \cup \mathrm{C}_{0-2 h}\right)$ (per protocol data)

(Mean absolute and percentage difference from control; mean values +/- SEM)

\begin{tabular}{|c|c|c|c|c|}
\hline & \multicolumn{4}{|c|}{ Insulin tAUC $_{0-2 h}$ (control: $\left.3143.02 \mu \mathrm{IU} / \mathrm{l} . \mathrm{min}\right)$} \\
\hline & \multicolumn{2}{|c|}{ Absolute ( $\mu$ IU/I.min) } & \multicolumn{2}{|c|}{ Difference $(\%)$} \\
\hline & Mean & SEM & Mean & SEM \\
\hline \multicolumn{5}{|l|}{ Flatbread compositions } \\
\hline $80 \mathrm{~g} \mathrm{HFF}+15 \mathrm{~g} \mathrm{CPF}+2 \mathrm{~g} \mathrm{GG}+3 \mathrm{~g} \mathrm{BF}$ & $-875.42^{\star *}$ & 143.91 & $-27.85^{\star \star}$ & 4.58 \\
\hline $77 \mathrm{~g} \mathrm{HFF}+15 \mathrm{~g} \mathrm{CPF}+3 \mathrm{~g} \mathrm{GG}+5 \mathrm{~g} \mathrm{BF}$ & $-1117.30^{\star \star}$ & 143.73 & $-35.55^{\star \star}$ & 4.57 \\
\hline $81 \mathrm{~g} \mathrm{HFF}+15 \mathrm{~g} \mathrm{CPF}+4 \mathrm{~g} \mathrm{GG}$ & $-1398.20^{\star \star}$ & 141.79 & $-44.48^{\star \star}$ & 4.51 \\
\hline
\end{tabular}

HFF, high fibre; CPF, chickpea flour; GG, guar gum; BF, barley flour. Statistically significant: ${ }^{\star \star} P \leq 0.0001$. 
No reliable effect on self-reported appetite ratings was apparent for the fibre and CPF mixes used here or in our previous study ${ }^{(21)}$. Other research suggests that changes in plasma glucose $e^{(51,52)}$ or insulin per se $e^{(52,53)}$ may have limited effects on appetite. In addition, Clark \& Slavin ${ }^{(54)}$ concluded in a systematic review that most fibres do not reduce appetite in acute study designs. Nevertheless, there are several reports of enhanced satiety effects associated with the addition of legumes or specific fibres to foods and beverages ${ }^{(24,25)}$. Unfortunately, the levels of viscous fibres needed to influence appetite may be incompatible with desired sensory attributes of many products ${ }^{(5)}$.

Comparison of the PPG results with those from our previous study ${ }^{(21)}$ shows that the addition of $\mathrm{CPF}+4 \%$ GG reduced PPG by approximately $28 \%$ in this study and by approximately $35 \%$ in the previous study against slightly different control products; however, the effect here for addition of $2 \%$ GG (approximately $16 \%$ ) was much lower than previously observed (approximately 33\%). One of the key differences between the two studies is the use of venous blood here, in contrast to capillary blood in the previous study. Venous blood gives a lower PPG response compared with capillary blood (factor $0.67)^{(56,57)}$ and this was reflected in the absolute effect size for which the study was powered. The lower PPG values in this study could also be due to a better handling of the glycaemic carbohydrate load in this Indian population (e.g. higher insulin sensitivity), possibly related to the rather low BMI and age range of the subjects. Nevertheless, all the test mixes in the current study gave at least $15 \%$ reduction in PPG $+\mathrm{iAUC}$, an effect size that we believe to be a reasonable benchmark for a physiologically meaningful effect in a general population. Although this is a subjective judgement, there does not seem to be any threshold for beneficial effects of lowering PPG in reducing CVD risk ${ }^{(58)}$. Furthermore, there are intervention studies with the PPG-lowering drug acarbose indicating that this level of reduction is possible and linked to a more effective endogenous insulin secretion effect ${ }^{(59)}$.

While this research confirms the effect of specific, commercially feasible flour/fibre mixes in decreasing the PPG and PPI responses, further research should focus on the mechanism of action (MoA). One of the supposed MoA is a delayed entry of glucose in the systemic circulation originating from starch, largely due to the viscosity generated by GG in the gastrointestinal tract. However, the slow influx of glucose can only be determined by the dual ${ }^{(60)}$ or triple stable isotope technique ${ }^{(61)}$. In addition, incretin and glucagon measurements should be included in future studies to better characterise the overall nature of the physiological responses to these kinds of fibre and flour mixes.

\section{Conclusions}

Together with our previous research ${ }^{(21)}$ we have demonstrated that flatbread flour mixes incorporating a combination of GG and CPF can produce statistically significant reductions in PPG and PPI responses. The data suggest a dose-response effect at low levels of GG addition, although this would need to be confirmed. However, these additions do not appear to influence postprandial appetite or mood-related parameters. The results suggest that these additions to commercial flatbread flour mixes could be an efficacious and feasible approach to achieve reduced PPG and PPI responses to such starchy staples in both Caucasian and Southeast Asian populations.

\section{Acknowledgements}

The authors are grateful to Carole Verhoeven, Anne-Roos Hoogenraad and Anton Porcu (Unilever Clinicals, Vlaardingen) and to Tanvi Kadam and Anisha Pargal (Unilever Clinicals, Mumbai, India) for facilitating the clinical studies. The authors are also grateful to Ramitha K., Suman Majumder and Chandrika Mohanan and her design team at Unilever Bangalore for providing the flours and to Jack Seijen ten Hoorn for formulating the different flatbreads and to Lambda Therapeutic Research Ltd, Ahmedabad (India), for executing the clinical studies.

This research was entirely funded by Unilever.

H. M. B., K. M., M. A. M. S., P. M. and D. J. M. designed the research; M. A. M. S. and R. D. facilitated execution of the study; P. M. performed statistical analysis. H. M. B. wrote the manuscript with significant contributions from D. J. M., K. M., P. M. and R. D. H. M. B. and D. J. M. had primary responsibility for final content. All authors read and approved the final version of the manuscript.

All authors are employees of Unilever, which manufactures and markets consumer food products, including the flour used for the flatbreads in this study.

\section{Supplementary material}

For supplementary material/s referred to in this article, please visit https://doi.org/10.1017/S0007114517000277

\section{References}

1. Hu FB (2011) Globalization of diabetes. The role of diet, lifestyle and genes. Diabetes Care 34, 1249-1257.

2. International Diabetes Federation (2011) Guideline for management of postmeal glucose in diabetes. Brussels, Belgium: International Diabetes Federation. www.idf.org/sites/default/ files/postmeal\%20glucose\%20guidelines.pdf.

3. Wachters-Hagedoorn RE, Priebe MG, Heimweg JAJ, et al. (2007) Low-dose acarbose does not delay digestion of starch but reduces its bioavailability. Diabet Med $\mathbf{2 4}$, 600-606.

4. Chiasson JL, Josse RG, Gomis R, et al. (2002) Acarbose for prevention of type 2 diabetes mellitus: The STOP-NIDDM randomised trial. Lancet 359, 2072-2077.

5. Thomas D \& Elliott EJ (2009) Low glycaemic index, or low glycaemic load, diets for diabetes mellitus. The Cochrane Database of Systematic Reviews, issue 1, Art. No.: CD006296.

6. Blaak EE, Antoine JM, Benton D, et al. (2012) Impact of postprandial glycaemia on health and prevention of disease. Obes Rev 13, 923-984.

7. Van De Laar FA, Lucassen PLBJ, Akkermans RP, et al. (2006) Alpha-glucosidase inhibitors for people with impaired glucose tolerance or impaired fasting blood glucose. The Cochrane Database of Systematic Reviews, issue 4, Art. No. CD005061.

8. Schnell O, Mertes G \& Standl E (2007) Acarbose and metabolic control in patients with type 2 diabetes with newly initiated insulin therapy. Diabetes Obes Metab 9, 853-858. 
9. European Food Safety Authority Panel on Dietetic Products Nutrition and Allergies (2012) Guidance on the scientific requirements for health claims related to appetite ratings, weight management, and blood glucose concentrations. EFSA Journal 10, 2604.

10. Lafiandra D, Riccardi G \& Shewry PR (2014) Improving cereal grain carbohydrates for diet and health. J Cereal Sci 59, 312-326

11. Henry CJK \& Kaur B (2014) Diet-based management and treatment of diabetes. In World Clinics - Diabetologia - Type 2 Diabetes Mellitus, pp 1-19 [V Mohan and R Unnikrishnan, editors]. New Delhi: Jaypee Brothers Medical Publishers (P) Ltd.

12. Khawaja KI, Fatima A, Mian SA, et al. (2012) Glycaemic, insulin and ghrelin responses to traditional South Asian flatbreads in diabetic and healthy subjects. Br J Nutr $\mathbf{1 0 8}$, 1810-1817.

13. Jenkins DJA, Kendall CWC, Axelsen M, et al. (2000) Viscous and nonviscous fibres, nonabsorbable and low glycaemic index carbohydrates, blood lipids and coronary heart disease. Curr Opin Lipidol 11, 49-56.

14. Marciani L, Gowland PA, Spiller RC, et al. (2001) Effect of meal viscosity and nutrients on satiety, intragastric dilution, and emptying assessed by MRI. Am J Physiol Gastrointest Liver Physiol 280, G1227-G1233.

15. Woerle HJ, Albrecht M, Linke R, et al. (2008) Importance of changes in gastric emptying for postprandial plasma glucose fluxes in healthy humans. Am I Physiol Endocrinol Metab 294, E103-E109.

16. Blackburn NA, Redfern JS, Jarjis H, et al. (1984) The mechanism of action of guar gum in improving glucose tolerance in man. Clin Sci (Lond) 66, 329-336.

17. Edwards CA, Johnson IT \& Read NW (1988) Do viscous polysaccharides slow absorption by inhibiting diffusion or convection? Eur J Clin Nutr 42, 307-312.

18. Goni I \& Valentin-Gamazo C (2003) Chickpea flour ingredient slows glycemic response to pasta in healthy volunteers. Food Chem 81, 511-515.

19. Thondre PS \& Henry CJK (2009) High-molecular-weight barley beta-glucan in chapatis (unleavened Indian flatbread) lowers glycemic index. Nutr Res 29, 480-486.

20. Radhika G, Sumathi C, Ganesan A, et al. (2010) Glycaemic index of Indian flatbreads (rotis) prepared using whole wheat flour and atta mix-added whole wheat flour. BrJ Nutr $\mathbf{1 0 3}$ 1642-1647.

21. Boers HM, MacAulay K, Murray P, et al. (2016) Efficacy of different fibres and flour mixes in South-Asian flatbreads for reducing post-prandial glucose responses in healthy adults. Eur J Nutr (Epublication ahead of print version 21 June 2016).

22. Mohanan C, Nediyedath SK \& Shanmugam A (2013) Wheat flour composition. Publication number: US20130209648 A1. http://www.google.com/patents/US20130209648

23. Tosh SM (2013) Review of human studies investigating the post-prandial blood-glucose lowering ability of oat and barley food products. Eur J Clin Nutr 67, 310-317.

24. Wanders AJ, van den Borne JJGC, De GC, et al. (2011) Effects of dietary fibre on subjective appetite, energy intake and body weight: a systematic review of randomized controlled trials. Obes Rev 12, 724-739.

25. Li SS, Kendall CWC, De Souza RJ, et al. (2014) Dietary pulses, satiety and food intake: a systematic review and meta-analysis of acute feeding trials. Obesity 22, 1773-1780.

26. Blundell J, De GC, Hulshof T, et al. (2010) Appetite control: methodological aspects of the evaluation of foods. Obes Rev 11, 251-270.

27. Brennan CS (2005) Dietary fibre, glycaemic response, and diabetes. Mol Nutr Food Res 49, 560-570.
28. Ekstrom LM, Bjorck IM \& Ostman EM (2013) On the possibility to affect the course of glycaemia, insulinaemia, and perceived hunger/satiety to bread meals in healthy volunteers. Food Funct 4, 522-529.

29. Gatenby SJ, Ellis PR, Morgan LM, et al. (1996) Effect of partially depolymerized guar gum on acute metabolic variables in patients with non-insulin-dependent diabetes. Diabet Med 13, 358-364

30. Wolever TMS, Jenkins DJA, Nineham R, et al. (1979) Guar gum and reduction of post-prandial glycaemia: effect of incorporation into solid food, liquid food and both. Br J Nutr 41, 505-510.

31. Wolf BW, Wolever TMS, Lai CS, et al. (2003) Effects of a beverage containing an enzymatically induced-viscosity dietary fiber, with or without fructose, on the postprandial glycemic response to a high glycemic index food in humans. Eur J Clin Nutr 57, 1120-1127.

32. Slaughter SL, Ellis PR, Jackson EC, et al. (2002) The effect of guar galactomannan and water availability during hydrothermal processing on the hydrolysis of starch catalysed by pancreatic alpha-amylase. Biochim Biophys Acta 1571, 55-63.

33. Dhital S, Gidley MJ \& Warren FJ (2015) Inhibition of $\alpha$-amylase activity by cellulose: kinetic analysis and nutritional implications. Carbohydr Polym 123, 305-312.

34. Symons LJ \& Brennan CS (2004) The influence of (1-3) (1-4)beta-D-glucan-rich fractions from barley on the physicochemical properties and in vitro reducing sugar release of white wheat breads. J Food Sci 69, C463-C467.

35. Jukanti AK, Gaur PM, Gowda CLL, et al. (2012) Nutritional quality and health benefits of chickpea (Cicer arietinum L.): a review. Br J Nutr 108, S11-S26.

36. Nestel P, Cehun M \& Chronopoulos A (2004) Effects of longterm consumption and single meals of chickpeas on plasma glucose, insulin, and triacylglycerol concentrations. Am J Clin Nutr 79, 390-395.

37. Zafar TA, Al-Hassawi F, Al-Khulaifi F, et al. (2015) Organoleptic and glycemic properties of chickpea-wheat composite breads. J Food Sci Technol 52, 2256-2263.

38. Johnson SK, Thomas SJ \& Hall RS (2005) Palatability and glucose, insulin and satiety responses of chickpea flour and extruded chickpea flour bread eaten as part of a breakfast. Eur J Clin Nutr 59, 169-176.

39. Juntunen KS, Laaksonen DE, Autio K, et al. (2003) Structural differences between rye and wheat breads but not total fiber content may explain the lower postprandial insulin response to rye bread. Am J Clin Nutr 78, 957-964.

40. Juntunen KS, Niskanen LK, Liukkonen KH, et al. (2002) Postprandial glucose, insulin, and incretin responses to grain products in healthy subjects. Am J Clin Nutr 75, 254-262.

41. Kallio P, Kolehmainen M, Laaksonen DE, et al. (2008) Inflammation markers are modulated by responses to diets differing in postprandial insulin responses in individuals with the metabolic syndrome. Am J Clin Nutr 87, 1497-1503.

42. Ludwig DS (2002) The glycemic index: physiological mechanisms relating to obesity, diabetes, and cardiovascular disease. JAMA 287, 2414-2423.

43. Laaksonen DE, Toppinen LK, Juntunen KS, et al. (2005) Dietary carbohydrate modification enhances insulin secretion in persons with the metabolic syndrome. Am J Clin Nutr 82, $1218-1227$.

44. Fang FS, Tian H, Li CL, et al. (2011) Comparison of postprandial insulin and fasting insulin on the evaluation of cardiovascular risk factors. Zhongua Yi Xue Za Zhi 91, 946-949.

45. Greenfield JR, Samaras K, Chisholm DJ, et al. (2007) Effect of postprandial insulinemia and insulin resistance on measurement of arterial stiffness (augmentation index). Int J Cardiol 114, 50-56. 
46. Morgan LM, Flatt PR \& Marks V (1988) Nutrient regulation of the entero-insular axis and insulin secretion. Nutr Res Rev $\mathbf{1}$ 79-97.

47. Pilichiewicz AN, Chaikomin R, Brennan IM, et al. (2007) Load-dependent effects of duodenal glucose on glycemia, gastrointestinal hormones, antropyloroduodenal motility, and energy intake in healthy men. Am J Physiol Endocrinol Metab 293, E743-E753.

48. Wachters-Hagedoorn RE, Priebe MG, Heimweg JAJ, et al. (2006) The rate of intestinal glucose absorption is correlated with plasma glucose-dependent insulinotropic polypeptide concentrations in healthy men. J Nutr 136, 1511-1516.

49. Ellis PR, Dawoud FM \& Morris ER (1991) Blood glucose, plasma insulin and sensory responses to guar-containing wheat breads: effects of molecular weight and particle size of guar gum. Br J Nutr 66, 363-379.

50. Jarvi AE, Karlstrom BE, Granfeldt YE, et al. (1995) The influence of food structure on postprandial metabolism in patients with non-insulin-dependent diabetes mellitus. Am J Clin Nutr 61, 837-842.

51. Aston LM, Stokes CS \& Jebb SA (2008) No effect of a diet with a reduced glycaemic index on satiety, energy intake and body weight in overweight and obese women. Int J Obes (Lond) 32 , 160-165.

52. Peters HPF, Ravestein P, Van Der Hijden HTWM, et al. (2011) Effect of carbohydrate digestibility on appetite and its relationship to postprandial blood glucose and insulin levels. Eur J Clin Nutr 65, 47-54.
53. Wikarek T, Chudek J, Owczarek A, et al. (2014) Effect of dietary macronutrients on postprandial incretin hormone release and satiety in obese and normal-weight women. Br J Nutr 111, 236-246.

54. Clark MJ \& Slavin JL (2013) The effect of fiber on satiety and food intake: a systematic review. J Am Coll Nutr 32, 200-211.

55. Ekstrom LMNK, Bjorck IM \& Ostman EM (2016) An improved course of glycaemia after a bread based breakfast is associated with beneficial effects on acute and semi-acute markers of appetite. Food Funct 7, 1040-1047.

56. Kuwa K, Nakayama T, Hoshino T, et al. (2001) Relationships of glucose concentrations in capillary whole blood, venous whole blood and venous plasma. Clin Chim Acta 307, 187-192.

57. Larsson-Cohn U (1976) Differences between capillary and venous blood glucose during oral glucose tolerance tests. Scand J Clin Lab Invest 36, 805-808.

58. Ceriello A (2005) Postprandial hyperglycemia and diabetes complications: is it time to treat? Diabetes 54, 1-7.

59. Kageyama S, Nakamichi N, Sekino H, et al. (1997) Comparison of the effects of acarbose and voglibose in healthy subjects. Clin Ther 19, 720-729.

60. Eelderink C, Moerdijk-Poortvliet TCW, Wang H, et al. (2012) The glycemic response does not reflect the in vivo starch digestibility of fiber-rich wheat products in healthy men. J Nutr 142, 258-263.

61. Rizza RA, Toffolo G \& Cobelli C (2016) Accurate measurement of postprandial glucose turnover: why is it difficult and how can it be done (relatively) simply? Diabetes 65, 1133-1145. 